\title{
Single-Phase Measurement of the Output Impedance of the Four-Quadrant Cascaded H-Bridge Converter Cell Using Wideband Signals
}

\author{
M. Petkovic and D. Dujic
}

This material is posted here with permission of the IEEE. Such permission of the IEEE does not in any way imply IEEE endorsement of any of EPFL's products or services. Internal or personal use of this material is permitted. However, permission to reprint / republish this material for advertising or promotional purposes or for creating new collective works for resale or redistribution must be obtained from the IEEE by writing to pubs-permissions@ieee. org. By choosing to view this document, you agree to all provisions of the copyright laws protecting it. 


\title{
Single-Phase Measurement of the Output Impedance of the Four-Quadrant Cascaded H-Bridge Converter Cell Using Wideband Signals
}

\author{
Marko Petković and Dražen Dujić \\ Power Electronics Laboratory - PEL, EPFL \\ Route Cantonale 15 \\ CH-1015 Lausanne, Switzerland \\ Phone: +41216935625 \\ Email: marko.petkovic@epfl.ch; drazen.dujic@epfl.ch \\ URL: https://www.epfl.ch/labs/pel
}

\section{Keywords}

$\ll$ Impedance measurement $\gg, \ll$ Modelling $\gg, \ll$ Multilevel converters $\gg$.

\begin{abstract}
The development and integration of power electronics equipment and converters for medium voltage ac and dc application has created different subsystem interactions that require proper investigation, understanding, description and estimation of global system stability through impedance-admittance measurements and identification. Four-quadrant Cascaded H-Bridge topology features high output voltage resolution and high effective switching frequency which enables high-dynamic, high-fidelity voltage perturbation injection for medium voltage impedance and admittance measurement. While being a perturbation injection and a measurement instrument it also needs to assure that it does not cause instabilities in the device under test. Hence, a mechanism to determine the output impedance of a single cell of a Cascaded H-Bridge converter is needed. To validate the theoretical developments, a new method to measure the single-phase output impedance in the $d q$-frame is proposed.
\end{abstract}

\section{Introduction}

The increase in energy demand combined with the advances in the field of power electronics has incentivized the expansion of renewable energy system as well as extensive placement into service of power conversion devices. The interaction of different elements in the grid, on one side leads to the change in power network behaviour that is becoming increasingly complex and on the other side results in system instabilities [1], [2]. In order to overcome this issue and prevent future instabilities, an early identification and characterization of the present system and its future potential components would reduce the risk of encountering unpredictable behavior which would, in turn, provide a stable network with uninterrupted operation. Some of the criteria used to assess the stability are presented in [3] and they rely either on analysing the minor-loop gain or the passivity of the system. Whatever the criteria are, the knowledge of the impedance of the network being analysed is required.

Unfortunately, the problem of high-power and medium voltage (MV) impedance/admittance measurement and system identification had not yet been fully addressed. Measurements in MV application require equipment designed for operation at MV level and a possibility to inject perturbation into MV system, while at the same time having sufficiently wide bandwidth in order to characterize the unknown object over a wide frequency range. These requirements combined are not easily fulfilled at the MV level and thus the research performed in this field is scarce and the devices developed for that purpose are few. The MV impedance estimators developed so far have either limited bandwidth, up to $1 \mathrm{kHz}$ or comprise an output side transformer in order to step-up the voltage to the MV levels, which in turn also limits the 
bandwidth. Still, the demand for such equipment is increasing due to the need to assist the development of recent medium voltage dc (MVdc) and medium voltage ac (MVac) applications, grid integration of renewable energy sources and storage devices, energy transmission and distribution in the MV range [4]-[6] and assure safe integration with the existing apparatus.

The MV converters based on the Cascaded H-Bridge (CHB) fall into the category of devices capable of having sufficiently high voltage output and bandwidth for MV impedance/admittance measurements. A variation of such topology that makes use of an active input converter stage interfaced to a multi-winding transformer (MWT) is presented in Fig. 1. The topology consists of step-down MV-MWT with 15 secondary phase-shifted outputs permitting to stack up to 5 cells per phase and thus effectively increase the output voltage levels and switching frequency allowing higher frequency voltage perturbation injection and impedance measurement. As a matter of fact, the presence of the MWT on the input side is one of the advantages of this topology. Having the MWT at the input means that there is no need to have a step-up transformer on the output side to elevate the voltage to MV level. As a result, the output stage high-frequency bandwidth is not limited by the output transformer. The CHB cells outputs are interfaced to a three-phase $L C$-type filter on which the voltage output is controlled. A single cell of the CHB converter is presented in Fig. 2. It consists of an Active Front End (AFE) as the input conversion stage and an H-bridge (HB) inverter as the output conversion stage. It is taken as an assumption that the dynamics of a single-phase leg consisting of five cells can be represented by the dynamics of one cell, which is why the cell in Fig. 2 is represented with an $L C$-type filter at the output.

The initial study on the feasibility of this solution was performed in [7], where there was a need to superimpose a high-frequency small-signal in addition to the fundamental component. When this is performed in a closed-loop, there exist possible interactions of synchronization and closed control loops of AFE and HB. Our work seeks to reveal if the presence of an active input element, such as AFE, impacts the shape of the impedance of the output stage HB and subsequently the cascaded connection of multiple HBs.

The rest of the paper is organised as follows. Section II describes the main features of a single cell of the CHB converter. Section III provides the state-space model of the cell in the $d q$-frame. Section IV characterizes the source-affected dynamics of the cell output. Section V proposed a new method to measure the output impedance of a single-phase inverter in the $d q$-frame using wideband pseudo-random binary sequence (PRBS) signal perturbation injection. Finally, Section VII concludes the work.

\section{Cascaded H-Bridge Cell}

The MWT (cf. Fig. 1) supplies three-phase isolated voltages from its secondary, low-voltage, side to the cell. Isolated three-phase supply makes the cells and the dc-links largely independent from each other [8]. The input filter of the cell is the $L$-type filter with its parasitic resistance. The rectification of the input voltage is performed by the three-phase AFE, while the dc-link serves as an energy buffer from which the output stage, a single-phase $\mathrm{HB}$, is supposed to inject perturbation voltages into an unknown network or device under test (DUT). The AFE control system comprises a PLL that performs synchronisation, as it is essentially a grid-connected converter through the MWT. Apart from this, it also performs the cascaded control of the dc-link voltage through the input current control. On the output side the HB performs cascaded control of its output voltage through the control of the filter inductor current.

The knowledge of the cell output impedance is beneficial due to the fact that the CHB should stay passive as seen by the DUT and not violate the stability criteria [3]. The information of the output impedance in this work is obtained by modelling the converter cell and by measuring it using singlephase measurements in the $d q$-frame combined with the application of wideband signals. Moreover, the influence of the active input element on the shape of the output impedance is investigated in order to reveal whether the input and the output stage can be considered decoupled for any future analysis. 


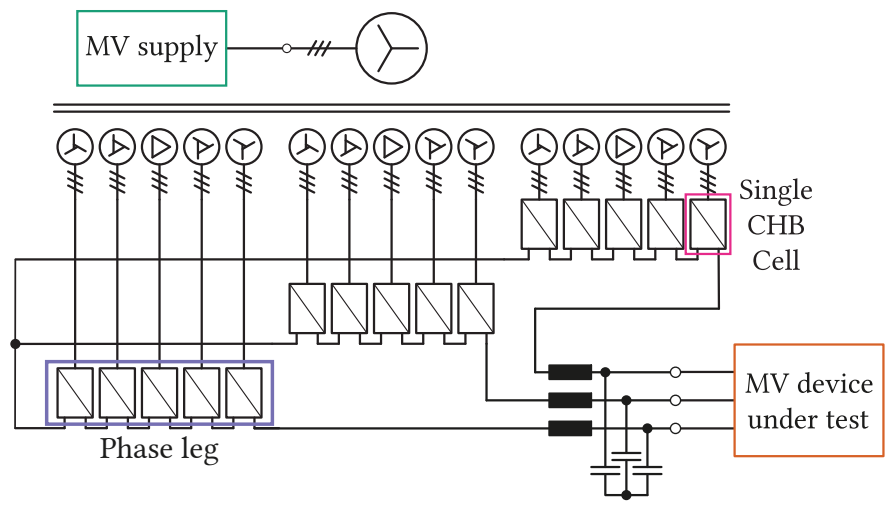

Fig. 1: Cascaded H-bridge topology for high dynamic medium-voltage perturbation injection and impedance measurement interfaced to a multi-winding transformer.

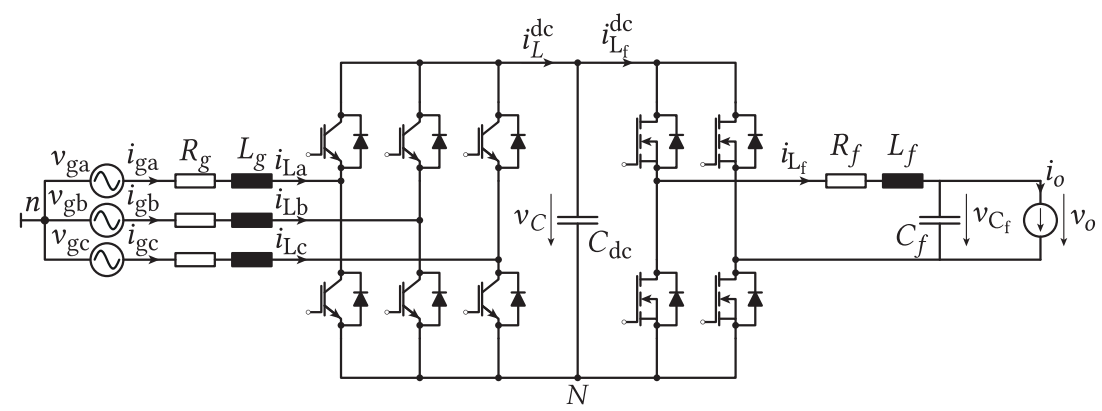

Fig. 2: CHB converter single cell comprising three-phase supplied Active Front End (AFE) interfaced to an $\mathrm{HB}$ phase inverter with an $L C$ filter.

\section{Single Cell Modelling}

\section{Active Front End Modelling}

Closed-loop modelling of the AFE follows the state-space modelling approach outlined in [9], [10] and the intermediate developments are not given in this work, but only the final open-loop and closedloop models in the Synchronous Reference Frame (SRF) are presented. State-space averaged model is presented in Eq. (1), where $V_{\mathrm{dc}}, D_{d}^{\mathrm{AFE}}, D_{q}^{\mathrm{AFE}}, I_{\mathrm{gd}}$ and $I_{\mathrm{gq}}$ are steady-state values of dc-link voltage with duty cycles and currents represented in $d q$-frame. To obtain the open-loop dynamics the model in Eq. (1) can be solved using Eq. (2) and the dynamics is represented by Eq. (3). State and output variables are given in the vector on the left hand side side, while the input variables are given in the right hand side vector of Eq. (3).

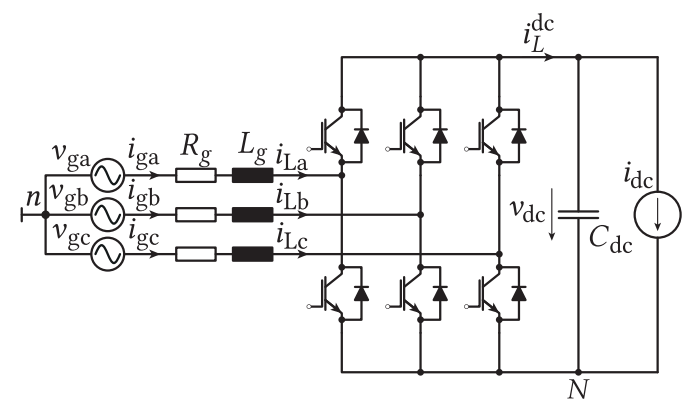

(a)

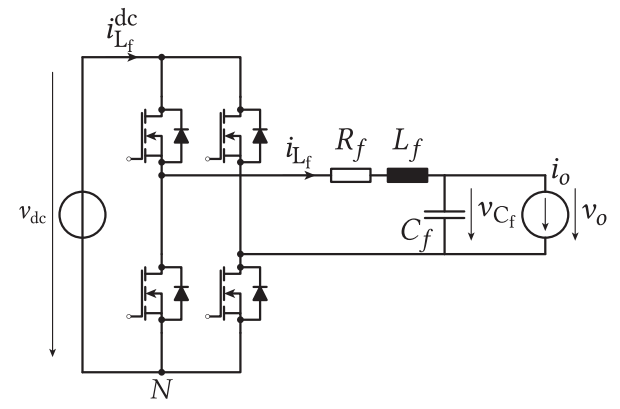

(b)

Fig. 3: Partitioned single cell of the CHB converter for the purposes of input and output stage modelling. 

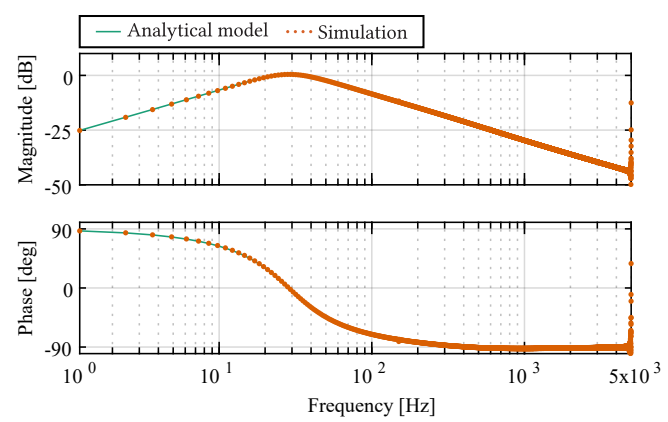

Fig. 4: Output impedance of the AFE under the input current and output voltage control loops closed.

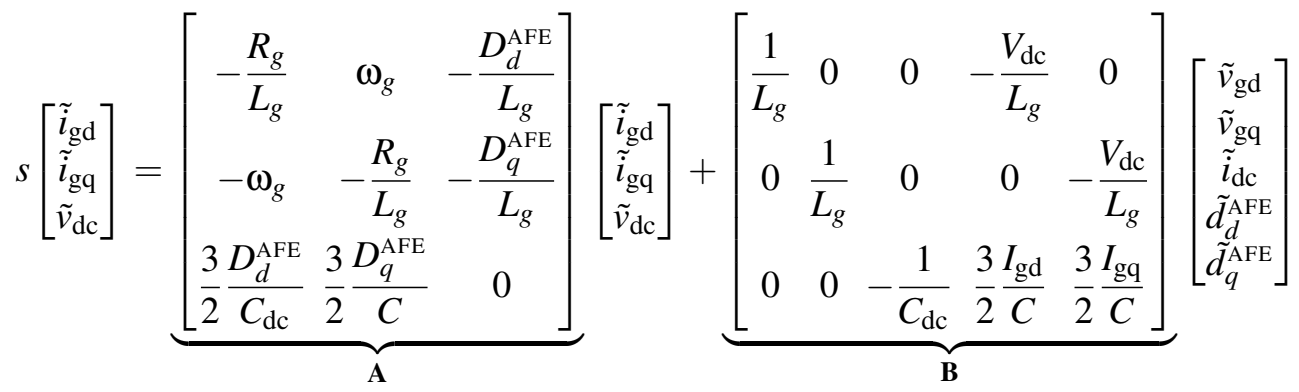

$$
\begin{aligned}
& {\left[\begin{array}{c}
\tilde{i}_{\mathrm{gd}} \\
\tilde{i}_{\mathrm{gq}} \\
\tilde{v}_{\mathrm{dc}}
\end{array}\right]=\underbrace{\left[\begin{array}{ccc}
1 & 0 & 0 \\
0 & 1 & 0 \\
0 & 0 & 1
\end{array}\right]}_{\mathbf{C}}\left[\begin{array}{c}
\tilde{i}_{\mathrm{gd}} \\
\tilde{i}_{\mathrm{gq}} \\
\tilde{v}_{\mathrm{dc}}
\end{array}\right]+\underbrace{\left[\begin{array}{ccccc}
0 & 0 & 0 & 0 & 0 \\
0 & 0 & 0 & 0 & 0 \\
0 & 0 & 0 & 0 & 0
\end{array}\right]}_{\mathbf{D}}\left[\begin{array}{c}
\tilde{v}_{\mathrm{gd}} \\
\tilde{v}_{\mathrm{gq}} \\
\tilde{i}_{\mathrm{dc}} \\
\tilde{d}_{d}^{\mathrm{AF}} \\
\tilde{d}_{q}^{\mathrm{AFE}}
\end{array}\right]} \\
& \mathbf{G}=\mathbf{C}(s \mathbf{I}-\mathbf{A})^{-1} \mathbf{B}+\mathbf{D} \quad \text { (2) } \quad\left[\begin{array}{c}
\tilde{\mathbf{i}}_{\mathrm{g}, \mathrm{dq}} \\
\tilde{v}_{\mathrm{dc}}
\end{array}\right]=\left[\begin{array}{ccc}
\mathbf{Y}_{\mathrm{in}, \mathrm{o}} & \mathbf{G}_{\mathrm{io}, \mathrm{o}} & \mathbf{G}_{\mathrm{ci}, \mathrm{o}} \\
\mathbf{T}_{\mathrm{oi}, \mathrm{o}} & -Z_{\mathrm{out}, \mathrm{o}} & \mathbf{G}_{\mathrm{co}, \mathrm{o}}
\end{array}\right]\left[\begin{array}{c}
\tilde{\mathbf{v}}_{\mathrm{g}, \mathrm{dq}} \\
\tilde{\tilde{\mathrm{d}}}_{\mathrm{dc}} \\
\tilde{\mathbf{d}}_{\mathrm{dq}}^{\mathrm{AFE}}
\end{array}\right]
\end{aligned}
$$

Direct link between the AFE and HB is the output impedance of the AFE and thus is the element that could influence the dynamics of the HB. For this reason the next important step is to find the closed-loop output impedance of the AFE. In the closed-loop the AFE is controlled in cascaded manner, with the inner loop being the input current control using proportional integral (PI) controllers in the SRF and the outer controller being the dc-link voltage PI controller. Under the current control loop closed, the output impedance is given as

$$
Z_{\mathrm{out}, \mathrm{cl}}^{\mathrm{gcc}}=-Z_{\mathrm{out}, \mathrm{o}}+\mathbf{G}_{\mathrm{co}, \mathrm{o}}\left(\mathbf{G}_{\mathrm{dec}}-\mathbf{G}_{\mathrm{PI}}^{\mathrm{i}}\right) \mathbf{G}_{\mathrm{io}, \mathrm{cl}}^{\mathrm{gcc}}
$$

Closing the dc-link voltage control loop reshapes the output impedance as

$$
Z_{\mathrm{out}, \mathrm{cl}}^{\mathrm{dvc}}=-\left[\mathbf{I}+\mathbf{G}_{\mathrm{co}, \mathrm{cl}}^{\mathrm{gcc}} \mathbf{G}_{\mathrm{PI}}^{\mathrm{v}}\right]^{-1} Z_{\mathrm{out}, \mathrm{cl}}^{\mathrm{gcc}}
$$

where $\mathbf{G}_{\mathrm{dec}}, \mathbf{G}_{\mathrm{PI}}^{\mathrm{i}}$ and $\mathbf{G}_{\mathrm{PI}}^{\mathrm{v}}$ are the current controller decoupling matrix, current controller PI regulator and voltage controller PI regulator. AFE with parameters in Table I was simulated in PLECS and its analytical output impedance from Eq. (5) was compared to the impedance obtained from the frequency response. The result is presented in Fig. 4 and it shows good match. Several things can be noted in the impedance shape. The impedance magnitude has a low value in low frequency range due to a high gain of the PI controller in the same region. The breaking point at $35 \mathrm{~Hz}$ where the impedance has almost unity gain denotes the end of the closed-loop dc-link control bandwidth. 


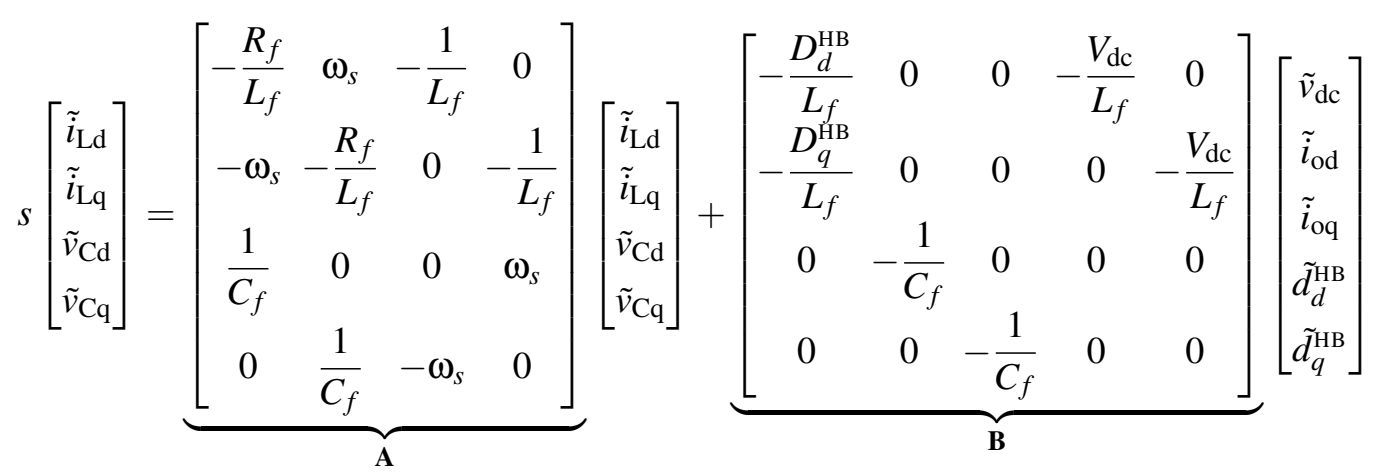

$$
\left[\begin{array}{c}
\tilde{i}_{\mathrm{Ld}} \\
\tilde{i}_{\mathrm{Lq}} \\
\tilde{v}_{\mathrm{Cd}} \\
\tilde{v}_{\mathrm{Cq}} \\
\tilde{i}_{L}^{\mathrm{dc}}
\end{array}\right]=\underbrace{\left[\begin{array}{cccc}
1 & 0 & 0 & 0 \\
0 & 1 & 0 & 0 \\
0 & 0 & 1 & 0 \\
0 & 0 & 0 & 1 \\
D_{d}^{\mathrm{HB}} & D_{q}^{\mathrm{HB}} & 0 & 0
\end{array}\right]}_{\mathbf{C}}\left[\begin{array}{c}
\tilde{i}_{\mathrm{Ld}} \\
\tilde{i}_{\mathrm{Lq}} \\
\tilde{v}_{\mathrm{Cd}} \\
\tilde{v}_{\mathrm{Cq}}
\end{array}\right]+\underbrace{\left[\begin{array}{ccccc}
0 & 0 & 0 & 0 & 0 \\
0 & 0 & 0 & 0 & 0 \\
0 & 0 & 0 & 0 & 0 \\
0 & 0 & 0 & 0 & 0 \\
0 & 0 & 0 & I_{\mathrm{Ld}} & I_{\mathrm{Lq}}
\end{array}\right]}_{\mathbf{D}}\left[\begin{array}{c}
\tilde{v}_{\mathrm{dc}} \\
\tilde{i}_{\mathrm{od}} \\
\tilde{i}_{\mathrm{oq}} \\
\tilde{d}_{d}^{\mathrm{HB}} \\
\tilde{d}_{q}^{\mathrm{HB}}
\end{array}\right]
$$

\begin{tabular}{ll}
\hline \hline$v_{\mathrm{g}, \mathrm{d}}=580 \mathrm{~V}$ & $f_{g}=50 \mathrm{~Hz}$ \\
$v_{\mathrm{g}, \mathrm{q}}=0 \mathrm{~V}$ & $L_{g}=5.4 \mathrm{mH}$ \\
$i_{\mathrm{g}, \mathrm{d}}=41.5 \mathrm{~A}$ & $R_{g}=10 \mathrm{~m} \Omega$ \\
$i_{\mathrm{g}, \mathrm{q}}=0 \mathrm{~A}$ & $v_{\mathrm{dc}}=1.2 \mathrm{kV}$ \\
$K_{\mathrm{p}, \mathrm{gcc}}=18$ & $K_{\mathrm{i}, \mathrm{gcc}}=33$ \\
$K_{\mathrm{p}, \mathrm{dvc}}=1.4$ & $K_{\mathrm{i}, \mathrm{dvc}}=192$ \\
\hline
\end{tabular}

Table I: AFE parameters

\begin{tabular}{ll}
\hline \hline$v_{\mathrm{C}, \mathrm{d}}=600 \mathrm{~V}$ & $f_{s}=50 \mathrm{~Hz}$ \\
$v_{\mathrm{C}, \mathrm{q}}=0 \mathrm{~V}$ & $L_{f}=1.4 \mathrm{mH}$ \\
$i_{\mathrm{o}, \mathrm{d}}=60 \mathrm{~A}$ & $R_{f}=100 \mathrm{~m} \Omega$ \\
$i_{\mathrm{o}, \mathrm{q}}=0 \mathrm{~A}$ & $C_{f}=24 \mu \mathrm{H}$ \\
$K_{\mathrm{p}, \mathrm{lcc}}=5$ & $K_{\mathrm{i}, \mathrm{lcc}}=5$ \\
$K_{\mathrm{p}, \mathrm{cvc}}=1$ & $K_{\mathrm{i}, \mathrm{cvc}}=52$ \\
\hline
\end{tabular}

Table II: HB parameters

\section{Single-Phase Inverter Modelling}

In order to find the closed-loop model, first the open-loop model and dynamics have to be defined and solved. As the dc-link voltage control is performed on the AFE side, the inverter input is initially considered to be an ideal voltage source (c.f. Fig. 3b). The modelling is performed in the $d q$-frame and it follows similar approach as the modelling of the AFE. The state-space averaged open-loop model is given in Eq. (6) whose open-loop dynamics is again solved using Eq. (2). The open-loop dynamics is represented with Eq. (7). The inverter control is performed as well in a cascaded manner in SRF. The inner loop controls the filter inductor current while the outer one controls the filter capacitor voltage, or the output voltage. Closed-loop output impedance under inductor current loop closed is given as Eq. (8). Closing the capacitor voltage control loop reshapes the impedance as presented in Eq. (9). The denominators $\Delta_{\mathrm{i}}$ and $\Delta_{\mathrm{v}}$ are calculated using Eqs. (10) and (11).

$$
\begin{aligned}
& {\left[\begin{array}{c}
\tilde{\mathbf{i}}_{\mathrm{L}, \mathrm{dq}} \\
\tilde{\mathbf{v}}_{\mathrm{C}, \mathrm{dq}} \\
\tilde{i}_{L}^{\mathrm{dc}}
\end{array}\right]=\left[\begin{array}{ccc}
\mathbf{G}_{\mathrm{iL}, \mathrm{o}} & \mathbf{T}_{\mathrm{oL}, \mathrm{o}} & \mathbf{G}_{\mathrm{cL}, \mathrm{o}} \\
\mathbf{G}_{\mathrm{iC}, \mathrm{o}} & \mathbf{Z}_{\mathrm{out}, \mathrm{o}} & \mathbf{G}_{\mathrm{co}, \mathrm{o}} \\
\mathbf{Y}_{\mathrm{in}, \mathrm{o}} & \mathbf{T}_{\mathrm{oi}, \mathrm{o}} & \mathbf{G}_{\mathrm{ci}, \mathrm{o}}
\end{array}\right]\left[\begin{array}{c}
\tilde{v}_{\mathrm{dc}} \\
\tilde{\mathbf{i}}_{\mathrm{o}, \mathrm{dq}} \\
\tilde{\mathbf{d}}_{\mathrm{dq}}^{\mathrm{HB}}
\end{array}\right]} \\
& \mathbf{Z}_{\mathrm{out}, \mathrm{cl}}^{\mathrm{lcc}}=-\mathbf{Z}_{\mathrm{out}, \mathrm{o}}+\mathbf{G}_{\mathrm{co}, \mathrm{o}} \Delta_{\mathrm{i}}^{-1}\left(\mathbf{G}_{\mathrm{dec}}^{\mathrm{i}}-\mathbf{G}_{\mathrm{PI}}^{\mathrm{i}}\right) \mathbf{T}_{\mathrm{oL}, \mathrm{o}}
\end{aligned}
$$

$$
\mathbf{Z}_{\text {out,cl }}^{\mathrm{cvc}}=-\Delta_{\mathrm{v}}{ }^{-1} \mathbf{Z}_{\text {out,cl }}^{\mathrm{lcc}}
$$




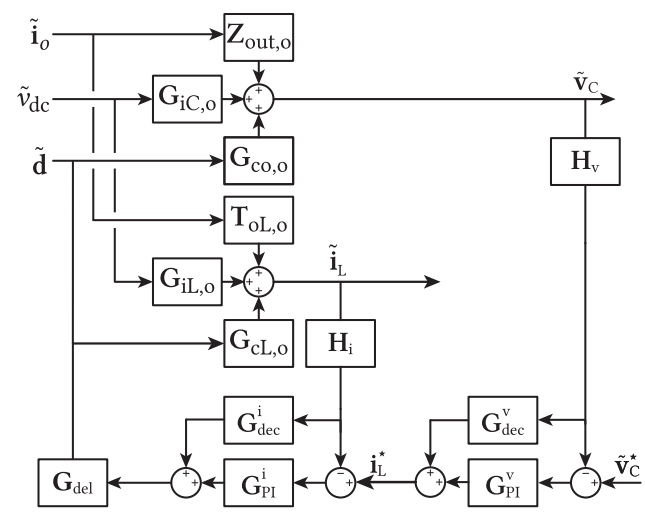

Fig. 5: Closed-loop dynamics of the HB inverter with cascaded inductor current and capacitor voltage control.

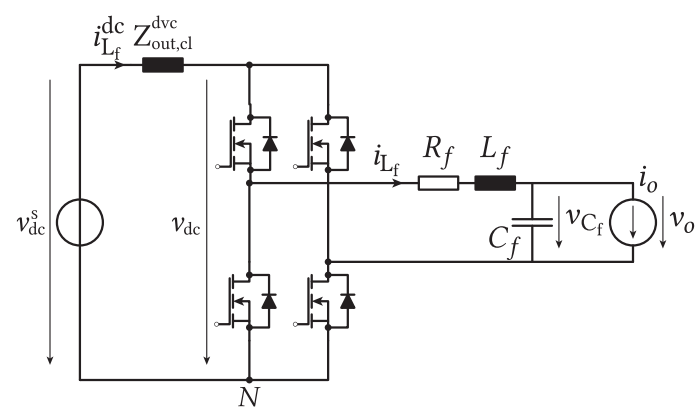

Fig. 6: HB with an output LC filter and internal source impedance.

$$
\Delta_{\mathrm{i}}=\left[\mathbf{I}-\mathbf{G}_{\mathrm{cL}, \mathrm{o}}\left(\mathbf{G}_{\mathrm{dec}}^{\mathrm{i}}-\mathbf{G}_{\mathrm{PI}}^{\mathrm{i}}\right)\right]^{-1} \quad(10) \quad \Delta_{\mathrm{V}}=\left[\mathbf{I}-\mathbf{G}_{\mathrm{co}, \mathrm{cl}}^{\mathrm{cvc}}\left(\mathbf{G}_{\mathrm{dec}}^{\mathrm{i}}-\mathbf{G}_{\mathrm{PI}}^{\mathrm{i}}\right)\right]^{-1}
$$

\section{Source-Affected Dynamics Characterization}

In previous section it was stated that the dc-link voltage source is ideal with respect to the fact that the voltage was controlled on the AFE side. However, in practice the control loops do not have an infinite bandwidth and as such the voltage source cannot be considered ideal. This means that the source contains a non-zero finite internal impedance which is the closed loop output impedance $Z_{\mathrm{out}, \mathrm{dl}}^{\mathrm{dvc}}$ of the active input element, i.e. the AFE. From Fig. 6, the dc-side current of the inverter can be defined as in Eq. (12). Replacing the open-loop dynamics from Eq. (7) with the closed-loop one, the dc-side current of the single-phase inerter, $i_{\mathrm{dc}}^{L}$, can be obtained using Eq. (13).

$$
i_{\mathrm{dc}}^{L}=\frac{v_{\mathrm{dc}}^{\mathrm{s}}-v_{\mathrm{dc}}}{Z_{\mathrm{out}, \mathrm{dl}}^{\mathrm{dvc}}}
$$

$$
i_{\mathrm{dc}}^{L}=Y_{\mathrm{in}, \mathrm{cl}}^{\mathrm{cvc}} v_{\mathrm{dc}}+\mathbf{G}_{\mathrm{ci}, \mathrm{cl}}^{\mathrm{cvc}} \tilde{\mathbf{v}}_{\mathrm{C}, \mathrm{dq}}^{\star}+\mathbf{T}_{\mathrm{oi}, \mathrm{cl}}^{\mathrm{crc}} \tilde{\mathbf{i}}_{\mathrm{o}, \mathrm{dq}}
$$

Furthermore, replacing Eq. (12) into Eq. (13) gives the $v_{\mathrm{dc}}^{\mathrm{s}}$ expressed in terms of $v_{\mathrm{dc}}$ as

$$
\tilde{v}_{\mathrm{dc}}=\frac{\tilde{v}_{\mathrm{dc}}^{\mathrm{s}}-Z_{\mathrm{out}, \mathrm{cl}}^{\mathrm{dvc}} \mathbf{G}_{\mathrm{ci}, \mathrm{cl}}^{\mathrm{cvc}} \tilde{\mathbf{v}}_{\mathrm{C}, \mathrm{dq}}^{\star}-Z_{\mathrm{out}, \mathrm{cl}}^{\mathrm{dvc}} \mathbf{T}_{\mathrm{oi}, \mathrm{cl}}^{\mathrm{crc}} \tilde{\mathbf{i}}_{\mathrm{odq}}}{1+Z_{\mathrm{out}, \mathrm{cl}}^{\mathrm{dvc}} Y_{\mathrm{in}, \mathrm{cl}}^{\mathrm{cvc}}}
$$

Replacing $\tilde{v}_{\text {dc }}$ in Eq. (7) by Eq. (14) gives the closed-loop source affected dynamics expressed Eq. (15). 

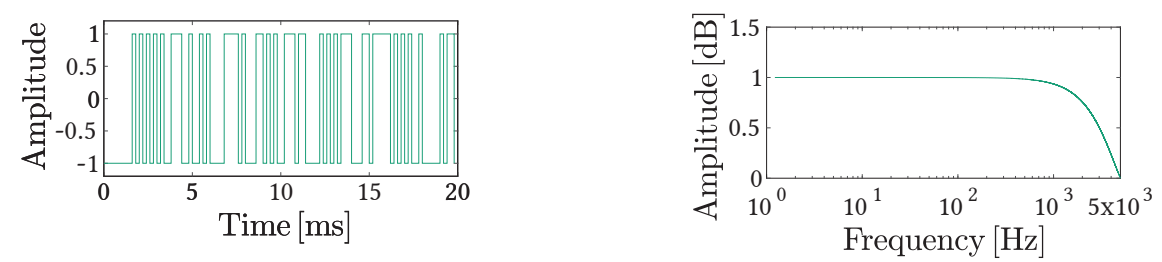

Fig. 7: PRBS-12 signal in time and frequency domain

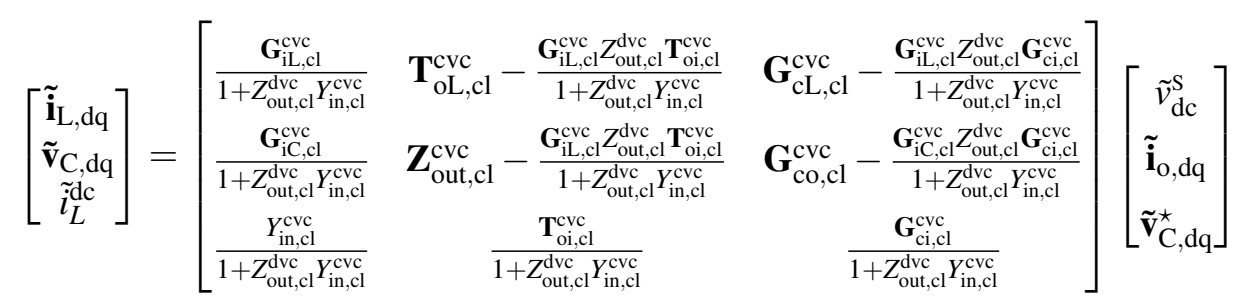

\section{Single Phase Measurement in $d q$-frame Using Wideband Signals}

Broadly used method in system identification measurements is the sine sweep method requiring one by one frequency small-signal perturbation injection and collection of responses, thus requiring large number of measurements and time to be executed. In the case where the measurement time is excessive, the measured system may change its operating point and subsequently the characteristics measured would change. This is improved to a degree with multi-tone signals but the energy of this signal is reduced with the number of tones it consists of. On the other hand, the PRBS signals (cf. Fig. 7) have lately been used substantially for system identification and impedance measurement [11]-[13]. The advantage of these signals lies in the fact that they are well suited for the characterization of dynamics systems where rapid measurement is necessary due to the possibility of variation of the system state in time. What is common for measurements in [11]-[13] is that they are performed in dc or three-phase ac systems after which the results are represented in $d q$-frame even though they represent ac measurements. Hilbert transform was successfully applied to single-phase measurement and representation in $d q$-frame in [14]. However, the transform was applied to a single tone perturbation and frequency sweep was performed. Here, a method that applies Hilbert transform to wideband PRBS signal is given. The reason for using the Hilbert transform is to create an orthogonal signal, containing all frequencies, to the one measured in single-phase. As the output impedance matrix in the $d q$-frame is defined as

$$
\mathbf{Z}_{\mathrm{out}, \mathrm{cl}}^{\mathrm{cvc}}=\left[\begin{array}{ll}
Z_{\mathrm{out}, \mathrm{cl}, \mathrm{dd}}^{\mathrm{cvc}} & Z_{\text {out,cl,qd }}^{\mathrm{cvc}} \\
Z_{\mathrm{out}, \mathrm{cl}, \mathrm{dq}}^{\mathrm{cc}} & Z_{\mathrm{out}, \mathrm{cl}, \mathrm{qq}}^{\mathrm{cvc}}
\end{array}\right]=\left[\begin{array}{ll}
\tilde{i}_{d d} & \tilde{i}_{d q} \\
\tilde{i}_{q d} & \tilde{i}_{q q}
\end{array}\right]^{-1}\left[\begin{array}{ll}
\tilde{v}_{d d} & \tilde{v}_{d q} \\
\tilde{v}_{q d} & \tilde{v}_{q q}
\end{array}\right]
$$

Where $\tilde{i}$ and $\tilde{v}$ are the small-signal current perturbation and the resulting current response. Measurement process of $\mathbf{Z}_{\text {out,cl }}^{\text {cvc }}$ requires four perturbation injections and four measurement sets. First perturbation is created in the $d q$-frame as $[p(t), 0]$ and then transformed into the $\alpha \beta$-frame using inverse Clarke transform. Afterwards only the $\alpha$-axis is injected into the current source in Fig. 3b, where the $p(t)$ is the PRBS signal in time domain. The resulting voltage measured would be equal to Eq. (17). If a second perturbation is created as $[-\hat{p}(t), 0]$ and injected in the same manner as before the resulting voltage leads to to Eq. (18).

$$
v_{C}^{s}=V_{m} \cos \omega_{s} t+v_{p}(t) \cos \omega_{s} t
$$

$$
v_{C}^{s}=V_{m} \cos \omega_{s} t-\hat{v}_{p}(t) \cos \omega_{s} t
$$

Applying the Hilbert transform to Eq. (18) and combining the resulting transformed equation with Eq. (17) provides two voltage measurements, one in $\alpha$-axis and one in $\beta$-axis as 


$$
v_{C, \alpha}^{s}=V_{m} \cos \omega_{s} t+v_{p}(t) \cos \omega_{s} t
$$

$$
v_{C, \beta}^{s}=V_{m} \sin \omega_{s} t+v_{p}(t) \sin \omega_{s} t
$$

Similarly, the third and fourth perturbations are created as $[0,-p(t)]$ and $[0,-\hat{p}(t)]$, respectively. After the injection the resulting voltages are obtained as

$$
v_{C}^{s}=V_{m} \cos \omega_{s} t+v_{p}(t) \sin \omega_{s} t
$$

$$
v_{C}^{s}=V_{m} \cos \omega_{s} t+\hat{v}_{p}(t) \sin \omega_{s} t
$$

If the Hilbert transform is now applied to Eq. (22) and the resulting equation is combined with Eq. (21) two voltage measurements, one in $\alpha$-axis and one in $\beta$-axis are obtained as

$$
v_{C, \alpha}^{s}=V_{m} \cos \omega_{s} t+v_{p}(t) \sin \omega_{s} t
$$

$$
v_{C, \beta}^{s}=V_{m} \sin \omega_{s} t-v_{p}(t) \cos \omega_{s} t
$$

Subsequently, Clarke transformation can be applied to Eqs. (19) and (20) and Eqs. (23) and (24) and the voltages can be represented in the $d q$-frame. Upon obtaining the measurements the frequency spectrum of the measurements is extracted using discrete Fourier Transform (DFT) algorithm from where Eq. (16) can be applied.

\section{Simulation Results}

The performance of the proposed modelling and measurement method is put under test using PLECS simulation environment. A $5 \mathrm{kHz}$ PRBS-12 signal is injected into on top of the fundamental load current and the resulting output voltage is measured. In order to investigate the effect of presence of an active converter stage in the cell, two types of CHB cell were simulated. The first cell type is a standalone HBinverter with an ideal voltage source as an input, while in the second case the AFE control system controls the capacitor dc-link voltage which serves as an input voltage source to the HB-inverter. In both cases, the HB-inverter is operating with parameters given in Table II. The $d$-axis closed-loop output impedances $\mathbf{Z}_{\text {out.cl }}^{\mathrm{cvc}}$ of the HB-inverter for with parameters in Table II are presented in Fig. 9. By comparing the two frequency responses it is easy to notice that the proposed modelling holds ground as the analytical characteristics matches the simulation results that are obtained by measuring the output impedance using the method proposed in this work. Besides, it is also noticeable that the output impedance of the HBinverter does not change when there is an active element on the input side.

\section{Conclusion}

This paper presented a promising solution for the problem of MV impedance/admittance measurement based on the MV-CHB converter. To confirm the suitability of the proposed idea, the model of the converter is developed systematically starting with the model of one single-phase cell of the converter and by confirming that the AFE input stage of a single cell does not affect the shape of the HB output impedance. The conclusion is reached through the wideband measurement of the output impedance using PRBS signals, while the results are shown methodically through analytical modelling and simulations. This type of measurement can successfully be performed in $d q$-frame by using the Hilbert transform of wideband signals. Compared to previous measurement methods, such as the much slower ac-sweep method, the wideband measurements allow rapid verification of concepts put forward. Moreover, the use of wideband measurements, namely the use of PRBS signals, reduce the risk of the measured system changing state while being measured. 

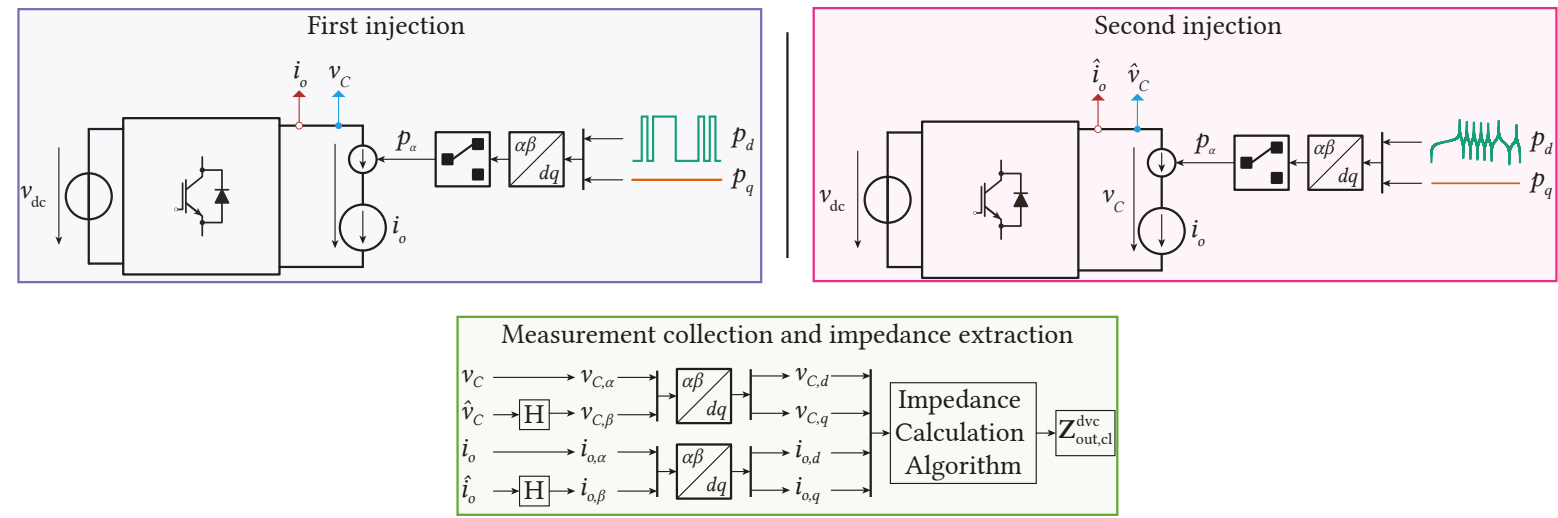

Fig. 8: Output impedance measurement of a single-phase inverter in the $d q$-frame

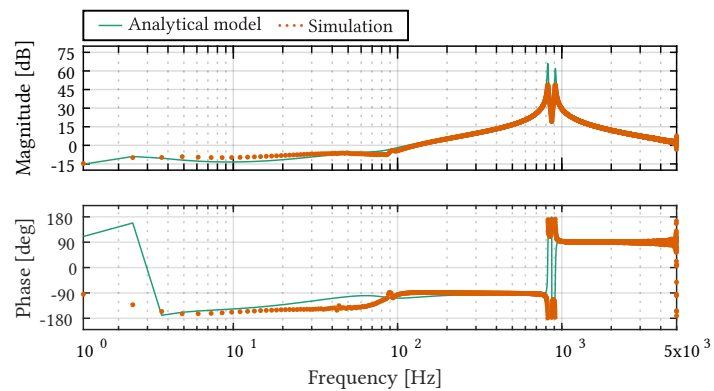

(a)

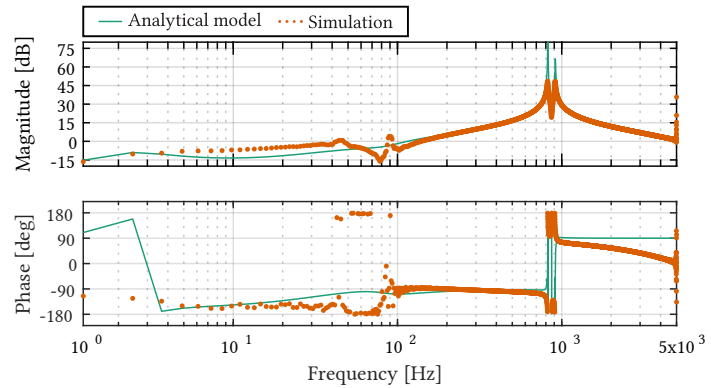

(b)

Fig. 9: $d$-axis output impedance $\mathbf{Z}_{\text {out.cl,dd }}^{\text {cvc }}$ with a) standalone-operation of the HB-inverter compared to the same characteristics, b) when the AFE is operating at the input stage.

\section{References}

[1] X. Wang and F. Blaabjerg, "Harmonic stability in power electronic based power systems: Concept, modeling, and analysis," IEEE Transactions on Smart Grid, pp. 1-1, 2018.

[2] U. Javaid, F. D. Freijedo, D. Dujic, and W. Van Der Merwe, "Dynamic assessment of source-load interactions in marine MVDC distribution," IEEE Transactions on Industrial Electronics, vol. 64, no. 6, pp. 4372-4381, 2017.

[3] A. Riccobono and E. Santi, "Comprehensive review of stability criteria for dc power distribution systems," IEEE Transactions on Industry Applications, vol. 50, no. 5, pp. 3525-3535, Sep. 2014.

[4] U. Javaid, F. D. Freijedo, W. van der Merwe, and D. Dujic, "Stability analysis of multi-port mvdc distribution networks for all-electric ships," IEEE Journal of Emerging and Selected Topics in Power Electronics, pp. 1-1, 2019.

[5] M. Mogorovic and D. Dujic, " $100 \mathrm{kw}, 10 \mathrm{khz}$ medium-frequency transformer design optimization and experimental verification," IEEE Transactions on Power Electronics, vol. 34, no. 2, pp. 16961708, Feb. 2019.

[6] M. Utvic, S. Milovanovic, and D. Dujic, "Flexible medium voltage dc source utilizing series connected modular multilevel converters," in [Detailed technical programme of the 21th European Conference on Power Electronics and Applications (EPE ECCE Europe)], 2019.

[7] M. Petković, N. Hildebrandt, F. D. Freijedo, and D. Dujić, "Cascaded H-Bridge Multilevel Converter for a High-Power Medium-Voltage Impedance-Admittance Measurement Unit," in 2018 International Symposium on Industrial Electronics (INDEL), Nov. 2018, pp. 1-8. 
[8] Y. Li, Y. Wang, R. Wang, J. Wu, H. Zhang, Y. Feng, S. Li, W. Yao, and B. Q. Li, "Distributed Generation Grid-Connected Converter Testing Device Based on Cascaded H-Bridge Topology," IEEE Transactions on Industrial Electronics, vol. 63, no. 4, pp. 2143-2154, Apr. 2016.

[9] R. W. Erickson and D. Maksimovic, Fundamentals of power electronics. Springer Science \& Business Media, 2007.

[10] T. Suntio, T. Messo, and J. Puukko, Power Electronic Converters: Dynamics and Control in Conventional and Renewable Energy Applications. Wiley, 2017.

[11] R. Luhtala, T. Roinila, and T. Messo, "Implementation of real-time impedance-based stability assessment of grid-connected systems using mimo-identification techniques," IEEE Transactions on Industry Applications, vol. 54, no. 5, pp. 5054-5063, Sep. 2018.

[12] T. Roinila, H. Abdollahi, S. Arrua, and E. Santi, "Real-time stability analysis and control of multiconverter systems by using mimo-identification techniques," IEEE Transactions on Power Electronics, vol. 34, no. 4, pp. 3948-3957, Apr. 2019.

[13] T. Roinila, T. Messo, R. Luhtala, R. Scharrenberg, E. C. W. de Jong, A. Fabian, and Y. Sun, "Hardware-in-the-loop methods for real-time frequency-response measurements of on-board power distribution systems," IEEE Transactions on Industrial Electronics, vol. 66, no. 7, pp. 5769-5777, Jul. 2019.

[14] Y. Liao, Z. Liu, H. Zhang, and B. Wen, "Low-frequency stability analysis of single-phase system with dq-frame impedance approach-part i: Impedance modeling and verification," IEEE Transactions on Industry Applications, vol. 54, no. 5, pp. 4999-5011, Sep. 2018. 